\title{
Experimental Campylobacter jejuni infection in the chicken: an animal model of axonal Guillain-Barré syndrome
}

\author{
C Y Li, P Xue, W Q Tian, R C Liu, C Yang
}

\begin{abstract}
Objective-To develop and characterise an animal model of paralytic neuropathy after Campylobacter jejuni infection. Campylobacter infection precedes development of many cases of Guillain-Barré syndrome and is particularly associated with cases having prominent axonal degeneration. Understanding the pathogenesis of Guillain-Barré syndrome after $C$ jejuni infection has been slowed by the lack of animal models.
\end{abstract}

Methods-A spontaneous paralytic neuropathy is described that developed in chickens from the farms of four patients with Guillain-Barré syndrome. The production of paralytic neuropathy in chickens experimentally fed Campylobacter jejuni isolated from one of these patients is reported. The sciatic nerves of the spontaneously paralysed chickens were examined pathologically in teased fibres, in plastic embedded sections, and by electron microscopy. Two large groups of chickens were then fed cultures of a $C$ jejuni (Penner type O:19) isolated from one of these patients.

Results-The chickens with spontaneous paralysis had pathologically noninflammatory neuropathy. Pathology in the sciatic nerves ranged from no detectable changes to severe Wallerianlike degeneration. In the experimentally inoculated groups, an average of $33 \%$ of the chickens became paralysed. The median time after inoculation to paralysis was 12 days. The lesions found in the first few days of paralysis included nodal lengthening and paranodal demyelination. In those animals that survived for several days after onset of weakness, the pathology was dominated by extensive Wallerian-like degeneration. Animals that survived for weeks with no clinically apparent neuropathy had paranodal remyelination in some teased nerve fibres, reflecting earlier paranodal demyelination.

Conclusion-Experimental inoculation with $C$ jejuni may provide a new model for understanding some forms of Guillain-Barré syndrome.

$(\mathcal{F}$ Neurol Neorosurg Psychiatry 1996;61:279-284)

Keywords: Guillain-Barré syndrome; Campylobacter jejuni; axonal degeneration
Campylobacter jejuni infection is increasingly recognised as an important antecedent of the Guillain-Barré syndromes. ${ }^{1-8} C$ jejuni can be cultured from stools from some patients with the disease, but in others the organism is probably cleared from the stools before onset of the neuropathy..$^{29-14}$ There is increasing interest in the possibility that glycoconjugate epitope(s) shared by the lipopolysaccharide of certain $C$ jejuni strains and by nerve may be the antigen(s) responsible for initiation of immune mediated nerve damage. ${ }^{11-14}$

Guillain-Barré syndrome as clinically diagnosed includes patients with inflammatory demyelinating changes ${ }^{15-17}$ and patients dominated pathologically by Wallerian-like axonal degeneration. ${ }^{16-19}$ Some patients with demyelinating Guillain-Barré syndrome have antecedent $C$ jejuni infection, ${ }^{720}$ but $C$ jejuni seems to be particularly associated with "axonal" forms of Guillain-Barré syndrome. ${ }^{6-8}$ This association applies to the nearly purely motor syndrome termed acute motor axonal neuropathy (AMAN) 16131618192122 that was recognised and characterised in studies of the summer epidemics that occur in northern China. ${ }^{1} 61723$

An important constraint on pathogenetic studies has been the absence of an animal model of neuropathy induced by $C$ jejuni. This report describes the occurrence of paralytic neuropathy in chickens in two settings. The first was a spontaneous disorder identified in the flocks of some farm families in which a member had recently developed GuillainBarré syndrome. The second was produced experimentally by feeding $C$ jejuni isolated from a patient with AMAN. These results may provide new models for the dissection of pathogenetic mechanisms in Guillain-Barré syndrome associated with $C$ jejun $i$ infection.

\section{Methods}

SPONTANEOUS-DISEASE GROUP

Patients admitted to the Second Teaching Hospital with Guillain-Barré syndrome are routinely questioned about similar disease in their villages or about sick animals on their farms. In the summer of 1993, the families of four patients gave a history of weak chickens developing near the time of onset of paralysis in the patient. Two of the authors travelled to the farms, and examined and purchased the chickens. Five chickens with spontaneously occurring disease were purchased from the farms of the four families, each of which had one member with Guillain-Barré syndrome. In all of the chickens, both the legs and the wings 
were involved. The sciatic nerves from these chickens were removed at necropsy and examined as described for the experimental groups below.

\section{EXPERIMENTAL GROUPS}

All birds in the experimental groups were inbred Esparpuk chickens, a local white feathered strain that resembles the Leghorn (AK Asbury, personal communication). All of the experimental birds were less than six months old. Half were males and half females. They were housed 15 to a cage; the control birds were caged in a separate region of the animal house. They were routinely raised by a local farm supplier, and were not from specific pathogen free, Marek's disease free, or avian leukosis free flocks. These birds were not cultured for $C$ jejuni before beginning the experimental studies, but subsequent studies have shown that $C$ jejuni can be cultured normally from nearly all such chickens.

Control group 1 consisted of four chickens that did not receive $C$ jejuni. Experimental group 1 consisted of 33 chickens that were fed $C$ jejuni by gavage, presumably into the gizzard, as described below. Experimental group 2 duplicated group 1 in a larger study of 66 chickens, all fed $C$ jejuni by the protocol described below.

STOOL CULTURE AND PREPARATION OF C $尹 E F U N I$ INOCULUM

The $C$ jejuni isolate used in all experiments was obtained from an eight year old boy (HB93-13) with AMAN (patient 4 in results). His stool was cultured by an enrichment method. Fresh stool was cultured in Preston Enriched Broth (Oxoid) in a microaerophilic $\left(5 \% \mathrm{O}_{2}, 3-11 \% \mathrm{CO}_{2}\right)$ environment at $41^{\circ} \mathrm{C}$. After 24 hours, the culture was inoculated on the selective CCDA plates and incubated under microaerophilic conditions at $41^{\circ} \mathrm{C}$ for 48 hours. The $C$ jejuni strain was typed as Penner O:19 by the USPHS Centers for Disease Control (Atlanta, GA, USA).

The $C$ jejuni was stored at $-70^{\circ} \mathrm{C}$ in brainheart infusion broth with $15 \%$ glycerol. The inocula were prepared by thawing the stored $C$ jejuni to room temperature. After immediate transfer to a CCDA plate, the sample was incubated in a microaerophilic environment for 48 hours at $37^{\circ} \mathrm{C}$. The bacteria were flushed with enrichment broth to a standard turbidity. The chickens in the experimental groups fed $C$ jejuni each received $5 \mathrm{ml}$ of the inoculum by gastric gavage. The gavage tube was of sufficient length to reach the gizzard.

\section{CLINICAL AND PATHOLOGICAL ANALYSIS}

Animals were assessed regularly for weakness of the hind limbs, difficulty standing or walking, wing droop, head drop, and difficulty feeding. In many animals, once weakness began it progressed very rapidly (see results) and they died on the day of onset. When possible, weak animals were allowed to survive for several days before being killed by exsanguination. The sciatic nerves of all of the control chickens and the affected experimental chickens, as well as some clinically unaffected experimental chickens, were removed. No other pathological specimens were obtained. Specimens were processed in three ways: (1) samples were fixed with $4 \%$ paraformaldehyde, stained with $4 \%$ osmium, softened in graded concentrations of glycerol, stored in $100 \%$ glycerol, and examined after teasing; (2) other paraformaldehyde fixed samples were embedded in paraffin for haematoxylin and eosin staining and for combined silver (modified Naumienko-Feigin stain)-luxol fast blue staining; and (3) samples were fixed by $5 \%$ glutaraldehyde, postfixed in osmium, embedded in Epon, and sectioned at $1 \mu \mathrm{m}$. Thin sections of selected glutaraldehyde fixed samples were examined by electron microscopy.

\section{Results}

\section{SPONTANEOUS DISEASE GROUP}

The table summarises the histories of the four patients whose families had weak chickens. Three of the four had the AMAN pattern of Guillain-Barré syndrome. All of the chickens that were examined were weak. All had $C$ jejuni cultured from their stools. One was typed (chicken No 3, table) and found to be Penner 019 Lior H36.

The pathological examination of these chickens was restricted to the sciatic nerves. The most severely affected was chicken No 3, obtained from the family of patient HB-93-4, an 11 year old girl with pronounced slowing of her nerve conduction velocities and with a clinical diagnosis of AIDP. This chicken had mild mononuclear infiltration in the sciatic nerve, with occasional fibres undergoing demyelination and numerous fibres undergoing Wallerian-like degeneration. The sciatic nerve from the other weak chicken from this farm was histologically normal.

Chicken No 1 from the farm of a 47 year old woman with AMAN had several teased fibres with definite lengthening of the nodes of Ranvier and mild paranodal demyelination with occasional remyelination (fig $1 \mathrm{~A}$ and $\mathrm{B}$ ).

Summaries of patients with paralysed chickens

\begin{tabular}{|c|c|c|c|c|c|c|}
\hline Designation & $\begin{array}{l}\text { Agel } \\
\text { Sex }\end{array}$ & $\begin{array}{l}\text { Antecedent } \\
\text { diarrhoea }\end{array}$ & $\begin{array}{l}\text { C jejuni } \\
\text { isolate }\end{array}$ & $\begin{array}{l}\text { Electro- } \\
\text { diagnostic } \\
\text { designation }\end{array}$ & $\begin{array}{l}\text { Paralysed } \\
\text { chickens } \\
\text { examined }\end{array}$ & Sciatic nerve pathology \\
\hline $\begin{array}{l}\text { HB93-2 } \\
\text { HB93-4 }\end{array}$ & $\begin{array}{l}47 \text { 우 } \\
119\end{array}$ & \pm & $\begin{array}{l}- \\
-\end{array}$ & $\begin{array}{l}\text { AMAN } \\
\text { AIDP }\end{array}$ & $\begin{array}{l}1 \text { (No 1) } \\
2(\text { No } 2, \text { No } 3)\end{array}$ & $\begin{array}{l}\text { Nodal lengthening, paranodal demyelination } \\
\text { No } 3 \text { had mild mononuclear inflammation, } \\
\text { mild internodal demyelination, and extensive } \\
\text { Wallerian-like degeneration } \\
\text { No } 2 \text { was normal }\end{array}$ \\
\hline $\begin{array}{l}\text { HB93-9 } \\
\text { HB93-13 }\end{array}$ & $\begin{array}{l}60^{\circ} \\
80^{\circ}\end{array}$ & - & $\overline{+}($ Penner O:19) & $\begin{array}{l}\text { AMAN } \\
\text { AMAN }\end{array}$ & $\begin{array}{l}1 \text { (No 4) } \\
1 \text { (No 5) }\end{array}$ & $\begin{array}{l}\text { Normal } \\
\text { Normal }\end{array}$ \\
\hline
\end{tabular}



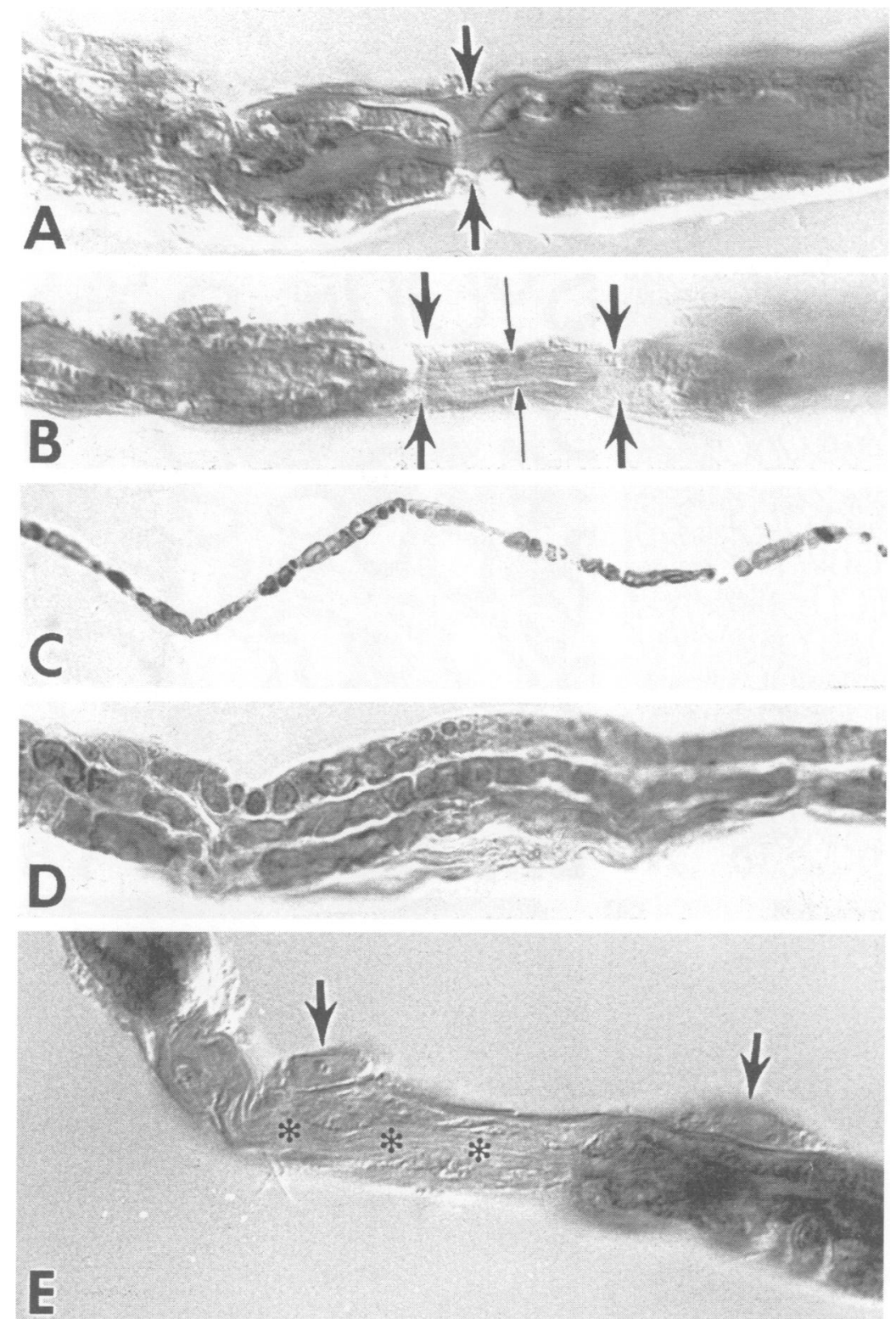

Figure 1 Osmicated teased nerve fibres. Panels $A, B$, and $E$ photographed with Nomarski optics. (A) Normal node of Ranvier (arrow) from a control chicken; original magnification $\times 1100$. (B) Paranodal demyelination and remyelination (chicken No 1) from the spontaneous disease group. Thin arrows identify the thin (remyelinated) myelin sheath in the former paranode. Thick black arrows identify the nodes; original magnification $\times$ 1100. (C) Fibre undergoing Wallerian-like degeneration from the same chicken; original magnification $\times$ 240. (D) Teased nerve fibres undergoing Wallerian-like degeneration from the most severely affected chicken in experimental group 1; original magnification $\times$ 680. (E) Paranodal demyelination in a chicken from experimental group 1. Demyelinated axon is identified by asterisks. Two mononuclear cells, presumably macrophages, are identified by arrows; original magnification $\times 1100$.

Figure 2 Increasing proportion of paralysed chickens with time in groups 1 and 2. The earliest animal (group 2) became weak on day 5 . The median time was day 12.

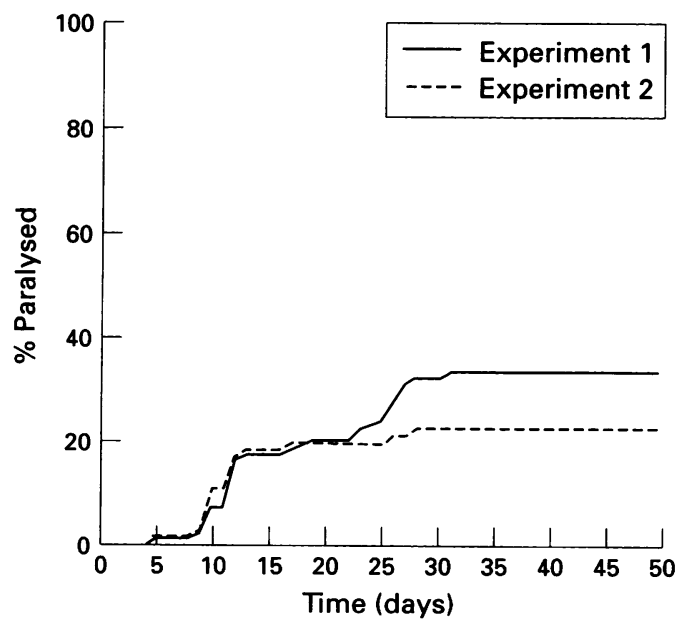

The weak chickens No 4 and 5 from the farms of children with the AMAN pattern had normal sciatic nerves.

\section{EXPERIMENTAL GROUPS}

The control chickens (those that were not fed $C$ jejuni) developed neither diarrhoea nor paralysis. They were killed on day 46 , and sciatic nerves were normal both by teased fibre preparations and by silver-LFB and haematoxylin and eosin stained paraffin sections. In particular, there was no paranodal demyelination or remyelination (fig 1A) and no evidence of Wallerian-like degeneration. Occasional mild paranodal changes were interpreted as preparative artifacts.

\section{Group 1}

The 33 chickens from group 1 all developed diarrhoea, beginning two to four days after feeding and lasting three to 14 days. $C$ jejuni was cultured from their stools. Penner typing of these cultures was not done.

Eighteen of these $33(55 \%)$ chickens became weak. Affected chickens were unable to stand, and some were paralysed. At early stages the chickens developed wing droop and head drop. Figure 2 shows cumulative plots of the times of onset of paralysis. The earliest onset of weakness (one chicken) was day 5 after inoculation. The median time to development of weakness was 12 days. Eleven of the 18 weak animals in group 1 were killed or died within a day of onset of weakness. The other seven were allowed to survive for up to 11 days after the onset.

Pathologically, five of the sciatic nerves from these 18 weak chickens were abnormal by examination of teased fibres in the mid-sciatic nerve. Three of these five were from animals that survived for 10 or 11 days after onset of weakness. The most severe abnormality was extensive ongoing Wallerian-like degeneration affecting about $25 \%$ of total fibres (figs 1D, $3 \mathrm{~A}-\mathrm{D})$ in a bird in which weakness became evident 18 days after giving $C$ jejuni. This bird died on day 19. The affected chickens also showed varying degrees of lengthening of the nodes of Ranvier and, in rare instances, paranodal demyelination (fig 1E). Mononuclear cells overlying these widened paranodes could occasionally be identified (fig 1E). Several fibres were identified by electron microscopy in which macrophages were present within the periaxonal space of affected internodes, separating the inner myelin lamellae from the axon, which was often degenerating (fig 3D).

The 15 clinically unaffected chickens from group 1 were all killed on day 46, and their sciatic nerves were examined by teased fibre studies. Only one nerve from these animals showed a few fibres undergoing late stages of Wallerian-like degeneration. Five of these 15 nerves had paranodal remyelination, with short intercalated internodes on one or both sides of the node. The proportion of fibres affected was never greater than $3 \%-4 \%$. 
Figure 3 Micrographs from the most severely affected chicken in

experimental group 1. (A) $A$ high proportion of fibres are undergoing Wallerianlike degeneration in this $1 \mu$ section embedded in plastic stained with toluidine blue; original magnification $\times 234$. (B) Higher power micrograph of this nerve illustrates, as well as numerous degenerating fibres, the presence of macrophages within the space formerly occupied by the axon (arrow); original

magnification $\times 738 .(C)$ Low power electron micrograph showing numerous fibres undergoing Wallerian-like

degeneration (examples identified by asterisks) with sparing of

unmyelinated fibres (examples identified within the boxes); original magnification $\times 2380$. (D) Degenerating axon (A) surrounded by a macrophage (M) in the periaxonal space; original magnification $\times 10000$
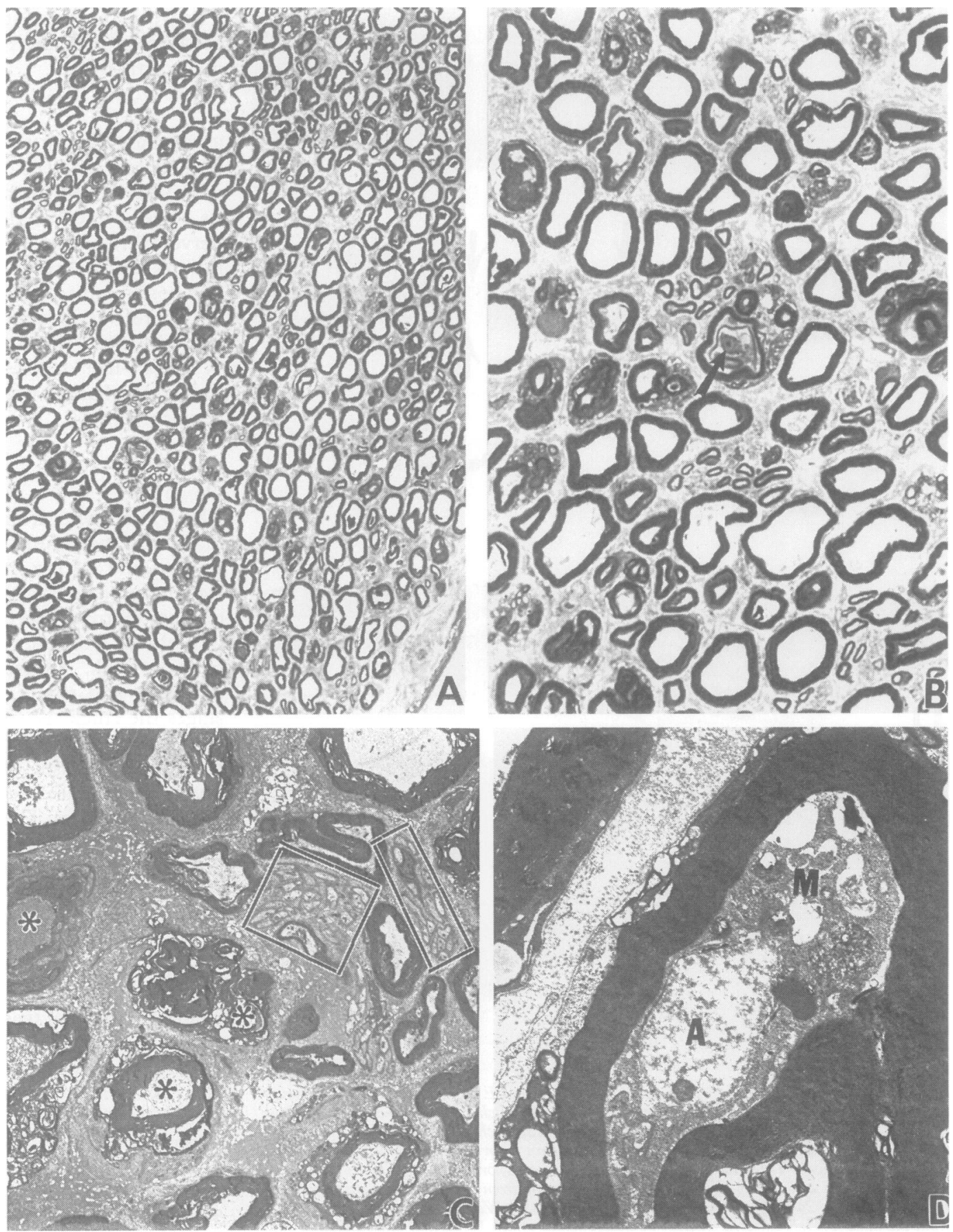

Group 2

Of the 66 chickens in group 2, $15(23 \%)$ developed paralysis. The earliest onset of weakness was on day 5 in two animals. The others became weak on days 9-26 (fig 2). Five of these 15 animals had sciatic nerve pathology by teased fibre preparations. Wallerian-like degeneration was found in two animals allowed to survive for several days after developing weakness. One of these chickens became weak on day 5 and was killed on day 12 (at a time when the animal was clinically improving). The other severely affected chicken became weak on day 17 and was killed on day 58. In addition to fibre loss and occasional fibres undergoing late stages of Wallerian-like degeneration, this chicken had extensive paranodal remyelination.
As in group 1 , most of the animals in group 2 that were killed on the day their weakness developed had normal or nearly normal sciatic nerves. The 41 animals that seemed clinically healthy were not examined pathologically.

In neither group were the mononuclear cell invasion or internodal demyelination characteristic of Marek's disease identified.

\section{Discussion}

This project began with the clinical discovery of paralysis in the chicken flocks of four families each of which had one member with GuillainBarré syndrome. The temporal association was striking: these families knew of no previous paralytic disease in their chicken flocks, nor of those within their villages. In addition, 
as discussed later, there were pathological similarities in two of these chickens with the pathology identified in necropsies of patients dying with the AMAN pattern of GuillainBarré syndrome. That $C$ jejuni might be the link between the human and chicken diseases was suggested by the culture of $C$ jejuni from the stools of one of the patients and by the fact that chickens are colonised by and excrete $C$ jejuni normally. We have confirmed that this applies to the flocks in Hebei Province by isolating $C$ jejuni from the culture of chicken faeces from provincial villages in which neither Guillain-Barré syndrome nor weak chickens were found (CY Li, TW Ho, R Liu, and I Nachamkin, unpublished data). Thus in these farm families, the spread of neuritogenic strains of $C$ jejuni from chickens to humans is a real possibility. ${ }^{6}$ However, because $C$ jejuni was successfully isolated from only one of these patients, it is not possible to compare the strains obtained from the chickens and patients. In the absence of such data, the simultaneous occurrence of human and spontaneous chicken disease can only provide a tantalising link. The experimental inoculation of chickens with the one successful stool isolation was undertaken to explore that possible link more rigorously.

The pathology in the most severely affected animal with spontaneous disease was dominated by Wallerian-like degeneration of sciatic nerve fibres. In addition, this animal and one other had evidence of nodal lengthening and paranodal demyelination. Strikingly, in three of the chickens there were no identifiable abnormalities in the sciatic nerves. Similarly, very minimal pathology has been noted in some patients dying with the AMAN pattern of Guillain-Barré syndrome, as well as in many of the chickens fed $C$ jejuni. The possible basis for this apparently anomalous finding is discussed later.

\section{EXPERIMENTAL FEEDING OF $C$ FE $Y U N I$}

The spontaneous disease led us to feed $C$ jejun $i$ isolated from a patient with AMAN (patient 4) to groups of experimental chickens. The two major findings in this experiment were, firstly, that most chickens fed $C$ jejuni by this protocol developed diarrhoea; and, secondly, that a proportion of these chickens developed paralytic neuropathy. Considering the two experimental groups together $33 \%$ of the animals developed weakness. Pathological studies confirmed a neuropathic basis for the weakness and indicated that some apparently healthy animals also had subclinical neuropathy, but also pointed out that very minimal or no changes could be present in the early stages of paralysis.

Several aspects of the experimental design will need to be altered in future studies. The strains of $C$ jejuni that the chickens were almost certainly excreting before inoculation should be determined. Because both the broth and the organisms contributed to the turbidity of the inoculum, we could not determine the number of organisms inoculated; determination of the size of the inoculum will need to be done by culture. Inoculation of a wider range of $C$ jejuni isolates is necessary. Finally, more extensive sampling of the PNS and CNS should be planned, including spinal roots and motor nerve terminals.

\section{PATHOLOGICAL CHANGES}

The pathological changes in paralysed chickens ranged from extensive Wallerian-like degeneration to no detectible abnormalities. Overall, the changes in these experimental birds had similarities to those seen in the AMAN pattern of Guillain-Barré syndrome in humans. ${ }^{1723}$ As in AMAN, ${ }^{17}$ lymphocytic infiltration was uncommon and scant (in the present study, the only chicken with identifiable lymphocytic infiltration of the sciatic nerve was No 3 in the spontaneous disease group). In AMAN, the mildest and probably the earliest change is nodal lengthening, which can occur with or without evidence of associated paranodal myelin damage. ${ }^{23}$ Lengthening and paranodal demyelination were seen in some of the experimentally fed animals killed early in the course of the disease, as well as in chickens No 1 and No 3 with spontaneous disease. In addition, among the experimental animals fed $C$ jejuni that did not develop clinical neuropathy and that were allowed to survive for nearly two months, some had evidence of paranodal remyelination. This change, never seen in control animals, presumably reflects remyelination in segments affected by earlier paranodal demyelination.

Wallerian-like degeneration was the dominant change in those animals that survived several days while weak. Wallerian-like degeneration is similarly the major finding in severe cases of AMAN. ${ }^{117}$ A provocative link between the disorders is the finding in the chickens of occasional fibres that had macrophages in the space normally occupied by the axon and inside otherwise normal appearing myelin sheaths. This is a very prominent change in the axon patterns of Guillain-Barré syndrome. ${ }^{161723}$ As described below, in AMAN this pattern has been linked to the presence of antibody and complement activation products in the periaxonal space.

A final pathological similarity is that, as in AMAN, many animals with paralysis had no detectable changes in the sciatic nerves. In some cases of AMAN, the changes in the spinal roots and sciatic nerves can be surprisingly mild in view of the severe (and fatal) paralysis. Similarly, AMAN paralysis can be rapidly reversible, ${ }^{6}$ indicating that some lesion milder than Wallerian-like degeneration must be present to allow early recovery. In AMAN, the possibilities of selective motor nerve terminal degeneration (TW Ho et al, unpublished data) or of antibody induced failure of impulse conduction (M Roberts, A Vincent, J Newsom-Davis, H Willison, TW Ho, unpublished data) are being considered. Similar considerations could apply to the chicken model described here. Motor nerve terminals were not examined. Alternatively, disease could be concentrated within the spinal roots, 
which were also unexamined in the present material.

Finally, the present data do not indicate the mechanism by which paralysis develops. Because the axonal patterns of Guillain-Barré syndrome are presumed to be immune mediated, an allergic reaction to some constituent of the organism, possibly the lipopolysaccharide, is attractive. However, the present data do not exclude the possibility of a neurotoxin produced by the organism. We have recently produced paralytic disease in one of eight rabbits immunised using complete Freund's adjuvant containing a lipopolysaccharide preparation from the same $C$ jejuni isolate used in these studies (CY Li, WQ Tian, P Xue, RC Liu, C Yang, unpublished data). The pathology was similar to that in axonal GuillainBarré syndrome and in the present study, including the presence of periaxonal macrophages. These results support an immune mediated mechanism. This work was supported by a grant from the Hebei Scientific
and Technology Committee, Hebei Province, PRC, and the and Technology Committee, Hebei Province, PRC, and the thank Drs Tony Ho, Guy McKhann, David Cornblath, Diane thank Drs Tony Ho, Guy McKhann, David Cornblath, Diane Irvin Nachamkin and Arthur Asbury (University of Pennsylvania) for helpful discussion. Dr John Griffin and Mr Rod Graham provided editorial suggestions and advice.

1 McKhann GM, Cornblath DR, Griffin JW, et al. Acute motor axonal neuropathy: a frequent cause of acute flaccid paralysis in China. Ann Neurol 1993;33:333-42.

2 Winer JB, Hughes RAC, Osmond C. A prospective study of acute idiopathic neuropathy. 2 . Antecedent events. $\mathcal{F}$ of acute idiopathic neuropathy. 2. Anteced

3 Vriesendorp FJ, Mishu B, Blaser M, Koski CL. Serum antibodies to GM1, peripheral nerve myelin, and Campylobacter jejuni in patients with Guillain-Barré syndrome and controls: correlation and prognosis. Ann Neurol 1993;34:130-5.

4 Kaldor J, Pritchard H, Serpell A, Metcalf W. Serum antibodies in Campylobacter enteritis. F Clin Microbiol 1983; 18:1-4.

5 Speed BR, Kaldor J, Watson J, et al. Campylobacter jejuni / Impulo Med f Aust 1987;147:13-6.
6 Ho TW, Mishu B, Li CY, et al. Guillain-Barré syndrome in northern China: relationship to Campylobacter jejuni
infection and anti-glycolipid antibodies. Brain 1995;118: infection and

7 Rees JH, Gregson NA, Hughes RAC. Anti-ganglioside GM1 antibodies in Guillain-Barré syndrome and their relationship to Campylobacter jejuni infection. Ann Neurol 1995;38:809-16.

8 Rees JH, Soudain SE, Gregson NA, Hughes RAC. Campylobacter jejuni infection and Guillain-Barré syndrome. N Engl f Med 1995;333:1374-9.

9 Dyck PJ. Is there an axonal variety of GBS? Neurology 1993;43:1277-80

10 Fujimoto S, Yuki N, Itoh T, Amako K. Specific serotype of Campylobacter jejuni associated with Guillain-Barré syndrome [letter]. F Infect Dis 1992;165:183.

11 Yuki N, Sato S, Fujimoto S, Yamada Y, Kinoshita A, Itoh T. Serotype of Campylobacter jejuni, HLA, and the G. Serotype of Campylobacter jejuni, HLA, and the

12 Yuki N, Handa S, Taki T, Kasama T, Takahashi M, Saito $K$. Cross-reactive antigen between nervous tissue and a bacterium elicits Guillain-Barré syndrome: molecular mimicry between gangliocide GM1 and lipopolysaccharide from Penner's serotype 19 of Campylobacter jejuni. Biomed Res 1992;13:451-3

13 Yuki N, Yoshino H, Sato S, Miyatake T. Acute axonal polyneuropathy associated with anti-GM $\mathrm{G}_{1}$ antibodies following Campylobacter jejuni enteritis. Neurology 1990;40 1900-2.

14 Kusunoki S, Chiba A, Kon K, et al. N-acetylgalactosaminyl GD1a is a target molecule for serum antibody in Guillain-Barré syndrome. Ann Neurol 1994;35:570-6.

15 Asbury AK, Arnason BG, Adams RD. The inflammatory lesion in idiopathic polyneuritis. Medicine 1969;48: 173-215.

16 Griffin JW, Li CY, Ho TW, et al. Pathology of the motorsensory axonal Guillain-Barré syndrome. Ann Neurol 1996;39:17-28.

17 Griffin JW, Li CY, Ho TW, et al. Guillain-Barré syndrome in northern China: the spectrum of neuropathologic changes in clinically defined cases. Brain 1995;118 577-95

18 Feasby TE, Gilbert JJ, Brown WF, et al. An acute axonal form of Guillain-Barré polyneuropathy. Brain 1986;109: 1115-26.

19 Feasby TE, Hahn AF, Brown WF, Bolton CF, Gilbert JJ, Koopman WJ. Severe axonal degeneration in acute Guillain-Barré syndrome: evidence of two different mechanisms? ₹ Neurol Sci 1993;116:185-92.

20 Enders U, Karch $\mathrm{H}$, Toyka KV, et al. The spectrum of immune responses to Campylobacter jejuni and glycoconimmune responses to Campylobacter jejuni and glycoconjugates in Guillain-Barre syndrome and in other neu-

21 Yuki N, Yoshino H, Sato S, Shinozawa K, Miyatake T. Severe acute axonal form of Guillain-Barré syndrome associated with IgG anti-GD $\mathrm{G}_{1 \mathrm{a}}$ antibodies. Muscle Nerve 1992;15:899-903.

22 McKhann GM, Cornblath DR, Ho TW, et al. Clinical and electrophysiological aspects of acute paralytic disease of children and young adults in northern China. Lance 1991;338:593-7.

23 Griffin JW, Li CY, Macko C, et al. Early nodal changes in Guillain-Barré syndrome. f Neurocytol 1996;25:33-51. 